\title{
Precariedad laboral universitaria con perspectiva de género a través de la IAP. El teatro INTERSOC como herramienta participativa de análisis en el aula
}

University precarious job with a gender perspective through the PAR. INTERSOC theater as a participatory analysis tool in the classroom

\author{
Stribor Kuric Kardelis \\ Universidad Complutense de Madrid, España \\ striborkuric@ucm.es \\ María José Díaz Santiago \\ Universidad Complutense de Madrid, España \\ mjdiaz@cps.ucm.es \\ Pablo López Calle \\ Universidad Complutense de Madrid, España \\ plopezca@cps.ucm.es \\ Irene Pastor Bustamante \\ Universidad Complutense de Madrid, España \\ aulateatrosocial@ucm.es
}

\begin{abstract}
Resumen: Con este artículo nos proponemos enlazar y contextualizar metodológicamente las técnicas desplegadas, en los últimos años, en el Aula Laboratorio de Teatro Social de la Universidad Complutense de Madrid y el proyecto docente Innovación metodológica del teatro social para el aprendizaje de las ciencias sociales (INSOCTEA), dentro del marco de las metodologías de Investigación-acción participativa (IAP). El teatro INTERSOC, como herramienta sociológica participativa diseñada para el aprendizaje y empoderamiento de la comunidad universitaria, parte de la elección colectiva del tema de análisis por parte del estudiantado, se continúa con el diseño colaborativo del espectáculo como forma de autoconocimiento y, posteriormente, se difunde como forma de reivindicación y transformación social.
\end{abstract}


Finalmente, se exponen someramente los principales resultados que se han observado desde el equipo de investigación en una de las obras de inteligencia común realizadas por el alumnado del Aula Laboratorio, desde la precariedad laboral y el género como propuesta escénica consensuada y recurrente anualmente como comunidad, pero de creación colectiva especificada en sus propias experiencias como grupo específico y que en su devolución, final, en el foro, a la comunidad universitaria muestra la colectivización de resistencias y empoderamientos en relación a la temática situada.

Palabras clave: precariedad laboral, género, Investigación-Acción-Participación, Teatro Social, docencia.

Abstract: With this article we intend to link and contextualize methodologically the techniques deployed in recent years in the Aula Laboratorio de Teatro Social de la Universidad Complutense de Madrid (Laboratory Classroom of Social Theater at the Complutense University of Madrid) and the teaching project Innovación metodológica del teatro social para el aprendizaje de las ciencias sociales (INSOCTEA) (Methodological innovation of social theater for the learning social sciences), within the framework of Participatory Action Research (Investigaciónacción participativa, IAP) methodologies. The INTERSOC theater, as a participatory sociological tool designed for the learning and empowerment of the university community, arises from the collective choice of the subject of analysis by the students, continues with the collaborative design of the show as a form of self-knowledge and, later, it is spread as a form of claiming and social transformation.

Finally, we expose the main results that have been observed by the research team in one of the common intelligence works carried out by the students of the Aula Laboratorio (Laboratory Classroom), from job insecurity and gender as scenic proposal that have been agreed and is recurrent every year as a community, but of collective creation specified in their own experiences as a specific group and that in their final return to the Forum, to the university community shows the collectivization of resistance and empowerment in relation to the situated theme.

Keywords: precarious job, gender, Participatory Action Research, Social Theater, teaching. 


\section{INTRODUCCIÓN}

El Teatro de Intervención Sociológica (en adelante Teatro INTERSOC), herramienta que se viene desarrollando en el Aula Laboratorio de Teatro Social de la Facultad de Ciencias Políticas y Sociología de la Universidad Complutense de Madrid ${ }^{1}$ y en las diferentes ediciones del proyecto de innovación docente denominado Innovación metodológica del teatro social para el aprendizaje de las ciencias sociales -INSOCTEA $^{2}$, muestra y pone en práctica un conjunto de técnicas para el análisis y la intervención en la realidad social. Técnicas basadas en la investigación y la dramatización del conflicto social, la participación de estudiantes y profesorado, y la implicación del público que asiste a la representación de las obras. En este sentido, el Teatro INTERSOC plantea, al mismo tiempo, un conjunto de interesantes problemas, de orden sociológico y epistemológico, acerca del teatro como instrumento de transformación social.

El teatro INTERSOC, que se nutre del denominado «teatro del oprimido», trabaja sobre el análisis y la representación de diferentes formas de dominación/opresión social (laboral, de género, de cultura, etc.), su carácter interseccional, así como de empoderamiento, desde una perspectiva fundamentalmente sociológica. Éste pretende mostrar, en cada representación, al «espectador y/o espectadora», un conjunto de personajes inmersos en relaciones de poder determinadas por la estructura social a la que pertenecen. Estos personajes se construyen, a partir de la categoría dialéctica-lógica del opresor-oprimido teorizada por Freire (1970 [2009]) y Boal $(1974,1982)$, que se acerca mucho al concepto de sujeto-sujetado que utiliza Jesús Ibáñez (1986), emparentado a su vez epistemológicamente con el modelo de estructurante-estructurado de Bourdieu (1973 [2002]). La formalización dialéctica de estos conceptos y categorías trata de problematizar en la práctica — es decir, en la trama que constituye la obra/pieza teatral—

${ }^{1}$ El Aula Laboratorio para su sostenibilidad ha contado con la financiación, los recursos materiales y los espacios de la Facultad de Ciencias Políticas y Sociología de la UCM, para la práctica teórica y práctica, y a la que estamos muy agradecidos por facilitar y promover la reflexión académica y cultural en el centro. Por otro lado, es necesario señalar que, además, en este curso académico, se ha ampliado la financiación con la concesión del proyecto «Innova-Docencia: INSOCTEA III, El Teatro Social como forma de investigación, acción participativa y aprendizaje de la Sociología del Trabajo y el género en el aula, 2019-2020», núm. 325, I.P. María José Díaz Santiago. Este artículo es uno de los productos derivados de la reflexión crítica e investigación promovidas dentro de este proyecto de creación académica colectiva.

2 Para ver la primera memoria del proyecto INSOCTEA I, puede visitar el siguiente enlace: https:// eprints.ucm.es/48356/ 
algunos de los límites o problemas epistemológicos centrales en el análisis científico de la realidad social y, en general, en la representación de la acción social: si las acciones dan cuenta de lo que el sujeto es o bien si el sujeto da cuenta de sus acciones (Pizzorno, 1987).

En la acción dramática construida por el alumnado participante en el Aula Laboratorio de Teatro Social y representada por el mismo alumnado, el poder de cada personaje sobre otros está determinado, o posibilitado, por la estructura social que a su vez los constriñe a través del poder/opresión que ejercen otros personajes. De manera que el orden social que determina la estructura de la obra se origina también en ellas y ellos mismos (construyen la trama al interactuar, al tomar decisiones), pero ellos y ellas son producto, a su vez, del orden social (de la trama y las decisiones que ya han sido escritas). La técnica empleada trata de ser, de este modo, una herramienta de "comprensión» de la acción social (v. g., comprender al otro), al mismo tiempo, de las dificultades para construir una ciencia social mediante la explicación de la misma, por ejemplo, al estilo del individualismo metodológico. Pero, en esta reflexión epistemológica sobre los límites del análisis de la realidad social para transformar la sociedad, se produce, en el teatro INTERSOC, una especie de metaacción —o se libera una suerte de pensamiento-acción de segundo orden - que da cuenta de una cierta transformación social, que consiste en mostrar y tomar consciencia de cómo dicho individualismo metodológico, característico del positivismo sociológico, opera con modelos de culpabilización y naturalización del destino de los personajes mediante la consideración de los motivos de los actores y actrices como las causas de sus acciones. En resumen, la comprensión colectiva del otro — es decir, la asunción de que el poder no se puede resistir-, exculparle de su destino, es un acto de transformación social.

Pues el teatro INTERSOC, a diferencia de otras formas de teatro social, trata de ir un poco más allá de la acción intelectual-comprensiva de la sociología crítica, en la medida en que añade al trabajo de identificación de las estructuras de dominación y de las formas en que son interiorizadas y reproducidas por el equipo actoral, la cuestión de la transformación. Si la pregunta por el cambio social, por la agencia, por el origen de la trama, es aquello que queda necesariamente excluido del pensamiento sociológico, el teatro INTERSOC propone al espectador y espectadora, en una segunda representación de la obra, la posibilidad de poder sustituir a diferentes actores/actrices y convertirse en el «autor» de una nueva trama encaminada a aliviar la opresión ejercida en la estructura del orden social. Esto es, ejercer la «resistencia» a la dominación, escribir la Historia... Es al tomar consciencia colectiva de la imposibilidad de dicha empresa en el interior de la obra cuando se produce la citada transformación social (Calderón y López Calle, 2014). El cambio social, en palabras de Benjamin, no puede ser represen- 
tado, sencillamente porque toda acción antecede a su representación (Benjamin 2012), remitiéndonos, con ello, a los debates en torno al llamado «giro lingüístico» que se produjo a finales del siglo XIX en el seno de las ciencias del espíritu cuando, según Foucault, alguien señaló al hombre como el sujeto-objeto imposible de la pregunta kantiana fundamental («¿quién habla?»). Las limitaciones de las ciencias sociales para representar la acción social, señaladas por aquel famoso giro, no han dejado de ser recordadas, frente al olvidadizo positivismo sociológico, por autores como el propio Foucault, Ibáñez, en nuestro país, o Derrida (2007), cuando afirman sin paliativos aquello de que no es posible decir el acontecimiento. Estos y otros autores han formado parte, de hecho, de un seminario de lecturas de carácter quincenal organizado por nuestro colectivo (INSOCTEA/Aula Laboratorio de Teatro Social UCM) sobre Teatro y Sociología ${ }^{3}$. Seminario realizado durante el curso 2018-19 en paralelo al desarrollo del taller de teatro INTERSOC en la Facultad de Ciencias Políticas y Sociología de la Universidad Complutense de Madrid.

A partir de estas premisas se comprende que precariedad laboral y desigualdad de género hayan sido las temáticas centrales en las diferentes y sucesivas propuestas del alumnado participante en el Aula Laboratorio de Teatro Social durante su andadura. Problemas sociales muy cercanos a las situaciones personales de los participantes, estudiantes de la Universidad Complutense de Madrid, pero además, factores estructurantes centrales de nuestro sistema social.

\section{EL TEATRO SOCIAL COMO HERRAMIENTA PARA LA IAP}

En este punto, consideramos fundamental dotar de un marco metodológico y epistemológico al trabajo desarrollado en el Aula Laboratorio de Teatro Social UCM. Desde sus inicios, la Investigación Acción Participativa (IAP) nos ha permitido desarrollar ese marco tan necesario para interpretar la información generada y situarla en un contexto de análisis sociológico ${ }^{4}$.

${ }^{3}$ Para ver la documentación trabajada durante el seminario, visítese https://insoctea.moodlecloud. com/

${ }^{4}$ En España, la tradición de la Investigación Acción Participativa se viene llevando a la práctica desde los años 80 y parte de la Escuela Cualitativista de Madrid, formada inicialmente por Jesús Ibáñez, Alfonso Ortí y Ángel de Lucas (Arias Fernández, 2016). Jesús Ibáñez (1986) desarrolla tres perspectivas de investigación: la distributiva, asociada a metodologías cuantitativas; la estructural, asociada a metodologías cualitativas; y la dialéctica, asociada a metodologías participativas. La IAP bebe de este último enfoque al basar- 
La aplicación de la perspectiva dialéctica, en términos de Ibáñez (1986), en la práctica, siempre es un tema complejo. Resulta imprescindible que las problemáticas e inquietudes trabajadas en el proyecto sean sentidas por la población afectada para que pueda situarse en el centro del proceso de investigación como protagonista y así poderlas comprender y transformar por sí mismas. En última instancia, se busca transformar la sociedad para volverla más justa a través del empoderamiento de colectivos para que puedan ser más críticos, conscientes y activos (Park, 1992). Se trata de un modelo ideal que inevitablemente pierde parte de su esencia al aplicarlo a la realidad social. A pesar de ello, existen múltiples técnicas mixtas y ejemplos prácticos de investigaciones que se han de desarrollado en los últimos años y que han sido nuestra referencia a la hora de llevar a cabo el proyecto del Aula Laboratorio. Algunos de estos modelos son el de la «sociopraxis», desarrollado por Tomás Villasante $(2006,2010)$ y la Red CIMAS, sobre todo aplicados en el campo del desarrollo comunitario, la aplicación de la IAP por el Colectivo IOE con asociaciones vecinales (Pereda, De Prada y Actis, 1997) o movimientos sociales (Pereda y De Prada, 2015) y la incorporación de la IAP en el análisis urbano (Francés Tortosa et al., 2016).

\section{¿Por qué es interesante incorporar elementos teatrales a la IAP?}

Utilizar elementos artísticos como el teatro aporta una serie de ventajas a la hora de plantear proyectos de investigación participativa o de intervención social (Madurga y Serra, 2016; Motos-Teruel y Benlliure, 2018). Las propuestas artísticas, por su condición de espacios simbólicos, paralelos y alternativos, permiten reconstruir y reimaginar la realidad social diseñando soluciones y alternativas desde conflictos imaginados venciendo pensamientos limitantes (López Fernández, 2011).

\footnotetext{
se en una ruptura con las asimetrías entre los agentes implicados de la investigación. Se trata de un enfoque que permite explorar la práctica sociológica como forma de transformación social, puesto que el objetivo de cualquier iniciativa que incorpore este enfoque es integrar la reflexión y la acción en un mismo proceso. De este modo, se fomenta la autogestión y la autonomía de los sujetos participantes para una posterior emancipación. Ibáñez no llega a desarrollar un manual metodológico concreto para llevar a la práctica el enfoque dialéctico, pero sí se refiere a este tipo de iniciativas como «socioanálisis». El término de Investigación Acción Participativa se origina en el ámbito de la sociología del trabajo, con el diseño de Elton Mayo (1945) y el desarrollo de Kurt Lewin (1947) de grupos de trabajo en fábricas dotados de mayor autonomía y autogestión para incrementar su productividad. En sus iniciativas se sienta el germen de lo que después en España recogería la Escuela Cualitativista de Madrid.
} 
Bajo el paraguas del concepto de «teatro social» se agrupa toda una serie de estilos, problemáticas y enfoques que, en última instancia, persiguen transformar la realidad, no solo representarla. Los ámbitos en los que se aplica este tipo de técnicas también es muy amplio. Algunos ejemplos son el desarrollo comunitario (Carnacea, 2013; Friedman, 2016; Morán, Ruiz y Marín, 2016; Vieites, 2016), el trabajo emocional y el desarrollo de la empatía para la mediación o con enfoques terapéuticos como el psicodrama (Castro y González, 2008; Castro y Martín, 2012; Hayes y Cantillon, 2014; Munro et al., 2015), o la construcción de herramientas pedagógicas (García-Huidobro, 2008; Preston, 2013; Vieites, 2013). En todos estos casos, la posibilidad de independizarse del lenguaje y las limitaciones que puede generar el texto escrito o la articulación de un argumento discursivo a la hora de abordar un problema pueden facilitar la capacidad de expresión de las personas que participan (Liamputtong y Rumbold, 2008). El teatro también permite cambiar el ángulo de visión ante un conflicto existente generando un juicio más consciente y atento que permita desenmarañar sus partes. Además, la creación colectiva facilita la elaboración de vínculos y espacios de escucha y diálogo en los que las personas que se encuentren en la misma situación se reconozcan y las que no, puedan empatizar con más facilidad (Hidalgo, 2011). De este modo, se multiplica el impacto de aquello que se desea transmitir y se potencia el empoderamiento grupal. En este tipo de proyectos se parte de la elaboración de una dramaturgia y de un planteamiento escénico de forma conjunta entre todas las personas participantes. Se despliega un proceso de ensayo-creación en el que la relación jerárquica con la figura del director en la posición más alta se rompe y se trata de aplicar una relación basada en la horizontalidad (Kuric, 2020: 228).

El «teatro del oprimido» de Augusto Boal (2012) es un tipo paradigmático de teatro social. En nuestros procesos participativos generados en el Aula Laboratorio de Teatro Social se emplea principalmente dos ramas del teatro del oprimido: el teatro imagen y el teatro foro, que facilitan a las personas participantes el proceso de convertirse en actores y actrices capaces de crear y re-crear escenas de la vida cotidiana. Este método teatral, como ya hemos introducido más arriba, se inspira en la «pedagogía del oprimido» de Paulo Freire (1970 [2009]), que rompe con las asimetrías entre educador y educado para crear una pedagogía inseparable de la comprensión y el análisis crítico de la realidad sociopolítica y económica. Augusto Boal parte de las figuras del opresor y el oprimido en una situación de conflicto para concretar historias a modo de expresiones genuinas y personales, en oposición a discursos dominantes e invitando a la denuncia y a la reflexión. El público abandona su rol pasivo y ya no delega sus acciones, pensamientos y sensaciones en los personajes, sino que los incorpora y transforma basándose en su propia realidad. Partiendo de los principios del teatro del oprimido, se han ido 
desarrollando muchas aproximaciones diferentes al teatro social y político como los ya mencionados «psicodrama», «teatro imagen», «teatro foro», «teatro de la escucha», «teatro encuentro», «teatro invisible», «teatro documental» o «teatro legislativo». Cada uno de estos enfoques tiene sus propias particularidades y técnicas, pero en todos los casos se busca un fin común: la reflexión y la transformación de la realidad a través del teatro (Vicente, 2013; Santos, 2017).

Desde el teatro INTERSOC hemos ido tomando diversos elementos de cada aproximación para componer el abanico de métodos que mejor se han ido ajustando a las necesidades de los temas trabajados y del alumnado que ha participado en el Aula Laboratorio de Teatro Social UCM. No obstante, nos hemos centrado en el teatro foro como eje principal del proceso creativo. Como veremos a continuación, este enfoque se ajusta perfectamente a los principios básicos de la Investigación Acción Participativa.

\section{¿Cómo hemos instrumentalizado el teatro desde la IAP?}

Las dimensiones básicas de una IAP son: la articulación de un sujeto colectivo, el consenso sobre la problemática y los objetivos, la extensión de la demanda inicial y la capacidad de transformación (Pereda, De Prada y Actis, 2003). Es importante tener en cuenta que no se trata de etapas sucesivas y lineales, que se van desarrollando de forma conjunta a lo largo de todo el proceso, sino que todas las dimensiones están interrelacionadas y no se pueden llevar a cabo de forma autónoma.

El sujeto colectivo incluye a todos los agentes que toman parte activa en los procesos de reflexión y toma de decisiones en el proyecto, siendo siempre las personas afectadas directamente por el tema trabajado las protagonistas, pero con representación de las redes económicas, políticas y culturales que se vinculan al problema. En el proyecto del Aula Laboratorio de Teatro Social el contexto trabajado es el académico, por lo que el sujeto colectivo parte de ese mismo ámbito. Se puede observar una representación gráfica de dicho sujeto colectivo en el Aula Laboratorio en la siguiente figura: 


\section{SUJETO COLECTIVO (En Proceso)}

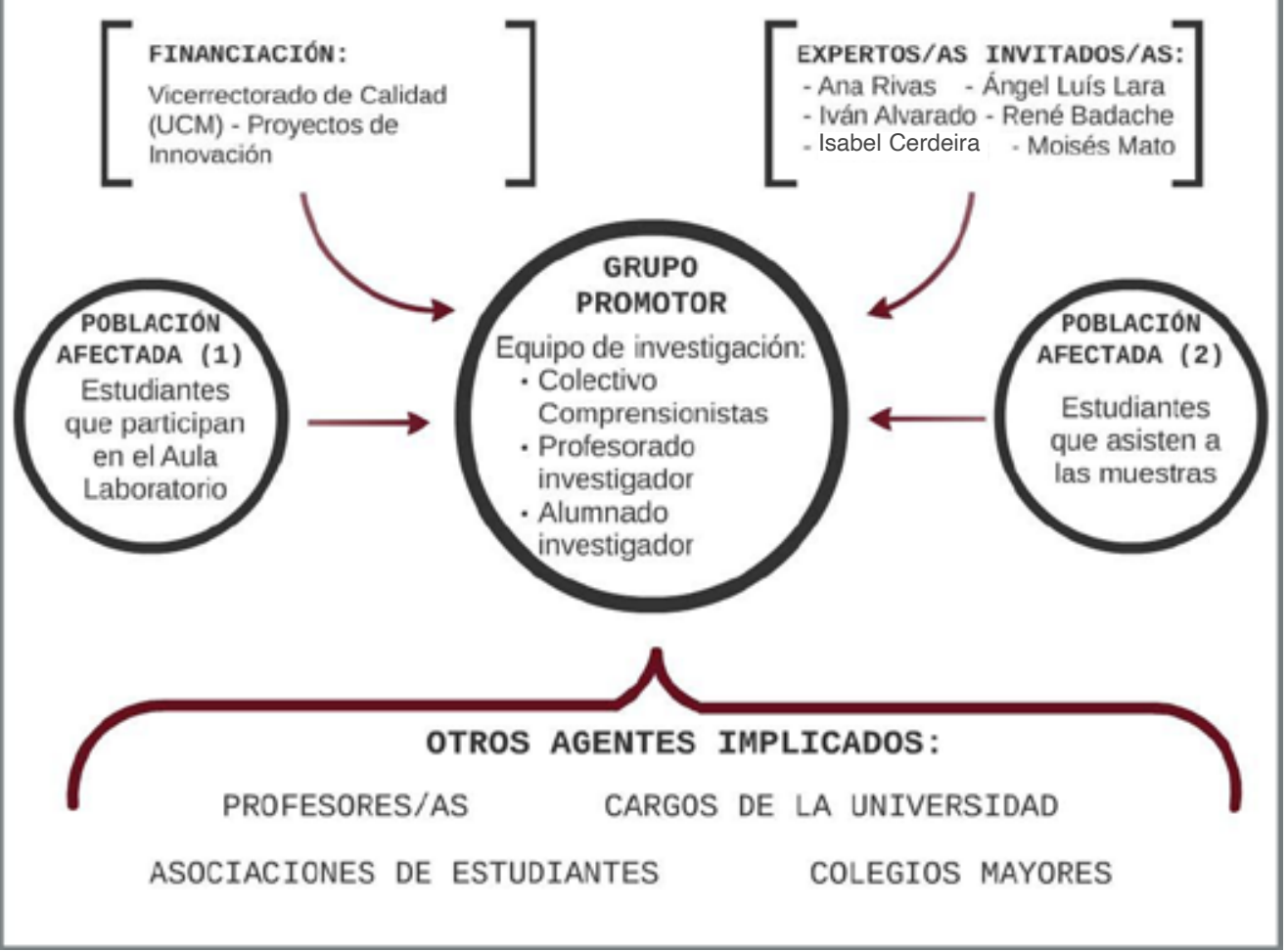

Fuente: Elaboración propia a partir del esquema utilizado por el Colectivo IOE en el «Proyecto +60», Investigación Acción Participativa en el barrio de Prosperidad de Madrid (Pereda, De Prada y Actis, 1997).

Como puede observarse, hemos subdividido al sujeto colectivo en cinco ámbitos principales:

- El grupo promotor se encuentra en el centro del modelo, es el núcleo más reducido y operativo y se ocupa de diseñar, impulsar y evaluar las tareas y la consecución de objetivos en el proyecto. Se compone del profesorado implicado, profesionales del Colectivo Comprensionistas (especializado en teatro social INTERSOC) y alumnado que se ha ido incorporando desde la primera edición del proyecto.

- La población afectada por la demanda inicial, alumnado que puede formar parte del proceso mediante dos vías: participando en el taller y en la creación colectiva 
de un espectáculo de teatro INTERSOC en el Aula Laboratorio o asistiendo a las muestras participativas del espectáculo realizado.

- La entidad financiadora es un elemento externo a la problemática, pero, a su vez, resulta fundamental a la hora de configurar el marco institucional y material para desarrollar el proyecto.

- El personal experto, profesionales en teatro INTERSOC y metodologías participativas que han acompañado en diversas ediciones y momentos del proceso asesorando y formando al grupo promotor.

- Otros agentes implicados, todas las personas, colectivos o instituciones que de forma directa o indirecta se ven afectados por la problemática trabajada en el proyecto. Personal universitario que es copartícipe del debate y la reflexión en las muestras, cargos institucionales que apoyan el proyecto, colegios mayores en los que se ha hecho alguna representación, etc.

La articulación del sujeto colectivo se va desarrollando a lo largo de todo el proyecto, por lo que se trata de un sujeto en proceso. En el Aula Laboratorio de Teatro Social, el sujeto colectivo se ha ido configurando a lo largo del periodo (2016-2020). Las problemáticas que se han ido trabajando se abordan como realidades completas y complejas que incluyen tanto elementos micro como macro, por lo que el sujeto colectivo se vuelve multidimensional. En este sentido, otra de las dimensiones básicas de la IAP es el consenso sobre la problemática y los objetivos a trabajar en el proceso: la demanda inicial. En nuestro caso, las temáticas abordadas han sido escogidas directamente por la población afectada, incrementando así la motivación a participar y contribuyendo a la continuidad del proyecto. Es fundamental para que pueda darse un proceso de IAP que la demanda inicial sea sentida por la población afectada (Pereda y De Prada, 2015). En nuestro caso, como exploraremos más adelante, se han trabajado principalmente las áreas de la precariedad laboral y las relaciones de género.

Las personas participantes en la investigación no son un grupo estanco que se mantiene a lo largo del proyecto, sino que se va adaptando y alterando con el objetivo de alcanzar un sujeto colectivo lo más amplio y representativo posible. Esta característica permite también hablar de extender la demanda inicial para maximizar la capacidad de análisis y transformación a través del juicio colectivo. El aspecto inherente de exhibición pública con el que cuentan las técnicas del Teatro Social (Madurga y Serra, 2016), y especialmente el teatro foro, permite que la extensión y difusión de la demanda inicial sea automática y se aumente el rango de acción a otras facultades y espacios en los que se concentren estudiantes y se puedan desarrollar las muestras. Con cada representación 
se genera un foro que permite implicar de forma continua a un mayor número de personas en el proceso de investigación-creación.

Es fundamental, en este punto, hacer referencia a la necesidad de horizontalidad y socialización efectiva del poder en el proceso de IAP. Resulta imprescindible aplicar un proceso permanente de diálogo y escucha entre los diferentes agentes del sujeto colectivo partiendo de la base de que el protagonismo y el poder ejecutivo resida en todo momento en la población afectada por la demanda inicial (Pereda, De Prada y Actis, 2003). Aquí reside otro aspecto en el que la incorporación del teatro social se vuelve crucial, el mismo proceso de creación colectiva facilita que la población afectada esté situada siempre en el eje central del proceso, asegurando que las expectativas del proyecto se mantengan abiertas a sus intereses y controlando que no se produzca una elitización en el Grupo Promotor.

\section{METODOLOGÍA DEL AULA LABORATORIO DE TEATRO SOCIAL EN LA DOCENCIA E INVESTIGACIÓN UNIVERSITARIA}

Uno de esos sujetos colectivos en proceso, que se ha designado población participante, está compuesto por personal actoral aficionado, que, en el caso del Aula Laboratorio, es el alumnado de ciencias sociales procedente, fundamentalmente, como se ha dicho, de la Facultad de Ciencias Políticas y Sociología de la UCM. Esta población estudiantil ha sido formada por profesionales expertos en precariedad laboral, género, socialización, teorías y aportaciones del teatro social, y en aspectos metodológicos sobre técnicas de investigación social, así como sus herramientas que les han ido dando los instrumentos pertinentes para el proceso de producción y acción social (Santos, 2017). A través de estos contenidos el alumnado participante, como población afectada, y también denominado espectactor o espectactriz, trabaja, fundamentalmente, las contradicciones esenciales en cualquier relación social. Dicho de otra manera, reflexionan

entre la explicación de la acción, de acuerdo a determinaciones estructurales que guían las acciones, como personajes, o bien de acuerdo a las oportunidades electivas que presentan los espectadores como autores de una nueva estructura social y que arrancaría de la problemática de Rousseau y D'Alembert acerca del teatro como instrumento de socialización ciudadana (De Mingo, 2012). De este modo se abre una nueva vía de investigación, para llegar a la objetividad, analizando en qué medida la subjetividad interviene en el proceso de construcción de conocimiento (Gaulejac, 2016), o poder (Foucault, 1999), 
para llegar a la compresión y búsqueda de soluciones a problemas sociales e intersubjetivos (Boal, 2004)» (Díaz y Pastor, 2018a: 41) .

Para ello, el alumnado del Aula Laboratorio aprende, por un lado, el uso de técnicas y herramientas de investigación social y, por otro, aprehenden en su revisión in situ la devolución en el foro. A través de la pluralidad metodológica, con base en la problemática o pregunta de investigación que ha generado el grupo, se desarrolla un proceso de devolución que se reactualiza con las aportaciones de una nueva población en el foro que, ahora convertida en espectactores y espectactrices, trabajan en común con el grupo motor desde la reflexión crítica, la comparación, la generación de analogías, etc. Proceso de deconstrucción sociológica que en la escena teatral muestra la gran riqueza de técnicas y herramientas con las que cuenta el proceso de creatividad social para acometer la complejidad social, representada y analizada en el foro. Dando cuenta que este hacer teatral e investigación sociológica se circunscribe en un contexto complejo, líquido, dinámico, cambiante y, en su devolución, también, susceptible de ser revisado por el método científico (Díaz y Pastor, 2018a y 2018b)

El acceso al proceso de investigación-acción se plantea a través de dos vías: la creación colectiva de un espectáculo de Teatro INTERSOC en el Aula Laboratorio y, de una forma menos continua pero con el mismo objetivo, la participación en las muestras del montaje con el posterior debate y reflexión colectiva.

En las primeras sesiones con el alumnado, una vez expuesta la estructura global de un proyecto de Teatro Social INTERSOC, se implementaron técnicas de diagnóstico participativo de necesidades para escoger las temáticas con las que más se identificaban. Principalmente mediante debates abiertos y compartiendo experiencias personales con el grupo se llegaron a definir tres ámbitos principales en los que se quería trabajar: el ecologismo y las posibilidades de agencia frente al medio ambiente, la precariedad económica del alumnado frente al incremento de tasas universitarias y las situaciones de acoso y discriminación que se observaban en la facultad. Una vez presentados, para escoger la temática central se implementó un ejercicio de Teatro Social basado en definir tres puntos en el aula de trabajo identificados con cada uno de los temas propuestos y después de caminar por el espacio durante unos minutos situarse más o menos cerca de los puntos definidos. Siguiendo este procedimiento se observó que la mayor parte de las personas que participaron se encontraban más vinculadas a la temática del género y con el tema de la precariedad en segundo lugar.

${ }^{5}$ Estas determinaciones se han estudiado en el seminario de Sociología del Teatro promovido dentro del proyecto INSOCTEA II, cuyo enlace a los materiales se ha incorporado anteriormente. 
El proceso de creación de cada pieza de teatro INTERSOC se realiza en tres partes: creación colectiva de la pieza, escenificación en el foro y análisis de la información recogida en todo el proceso investigador. La creación de la pieza realizada por los propios actores y actrices, convirtiéndose en los autores de dicha recreación colectiva y construida en varias fases: elección del hecho social a tratar, configuración de los papeles de los actores/actrices, consenso sobre el tipo de conflicto social a analizar, la dramaturgia, diseño escénico y contextualización de las realidades sociales a representar, puesta en escena y preparación de personajes para el diálogo en el foro.

De forma simplificada, los principales pasos que se han seguido a la hora de desarrollar reflexiones colectivas se han nutrido de la metodología del teatro foro de Augusto Boal, con aportaciones específicas del Teatro de la Escucha de Moisés Mato. Se ha comenzado por la definición de hechos paradigmáticos que reflejan las injusticias observadas, en relación con la problemática de trabajar y con experiencias personales de las personas participantes; a continuación se ha construido la cosmovisión de los posibles personajes y su posición en el hecho paradigmático como oprimidos, opresores u oprimidos-opresores; después se ha definido, con imágenes corporales, una situación de crisis que refleja el lado más decisivo del problema y, también con imágenes, se fijan las secuencias narrativas por las que pasará la historia hasta el momento de la crisis. Una vez establecido este marco, se pasa a improvisar en cada una de las secuencias y a diseñar la dramaturgia basándose en las improvisaciones y el debate posterior sobre las mismas. Antes de pasar a realizar la muestra, cada una de las personas que participan debe encarnar a distintos personajes incorporando su cosmovisión. De forma complementaria, en todo momento se han combinado los elementos prácticos de la investigación con un enfoque sociológico teórico sobre el género y la precariedad en el ámbito académico, definido por el equipo de investigación. Todos estos elementos han ayudado a profundizar en la temática escogida y a definir los elementos que se han ido trabajando a lo largo del proceso.

En este punto, expondremos brevemente el proceso de escenificación del foro. $\mathrm{La}$ pieza teatral, creada de forma colectiva, tiene aproximadamente veinte minutos de duración. Una vez ha sido representada una primera vez, se abre un debate moderado por una persona preparada para dinamizar una comunicación con el público, en el que se propone una segunda representación del mismo espectáculo, pero abierto a aportaciones/transformaciones dramatúrgicas diseñadas por el público participante en el foro. De esta manera se facilita una cocreación escénica en la que cualquiera puede intercambiarse por alguno de los personajes para cambiar algo de la historia con lo que no está de acuerdo. En cada una de las representaciones se aplica un juicio colectivo a partir de propuestas individuales en escena. Este dispositivo, basado en la exhi- 
bición incorporada de los resultados de la investigación, facilita en cada una de las muestras la extensión de la propuesta inicial a un ámbito cada vez más amplio y con una vocación de continuidad del proyecto a través de la comprensión de la realidad social y de la activación de la inteligencia colectiva del sujeto investigador como agencia.

\section{PRECARIEDAD LABORAL Y GÉNERO. INTELIGENCIA COLECTIVA EN EL AULA}

Como se ha ido diciendo en los párrafos anteriores, lo destacable es que el proceso de creación y de investigación tenga como punto de partida la elaboración de un texto construido, dialogado y colectivo que, a través del consenso, transforma al sujeto de investigación con las aportaciones de espectactores/rices. Proceso de trabajo que, a su vez, parte de la revisión documental, así como de sus propias biografías y subjetividades a través de la reflexión crítica y creación colectiva. La obra Master Vale Callar, que aquí analizaremos, es una pieza de Teatro INTERSOC que aborda la precariedad laboral desde el abuso de poder, el patriarcado y el acoso ambiental sexista en el ámbito universitario $^{6}$. La obra se fragua a través de una pluralidad de herramientas enmarcadas en la aproximación metodológica explicada en el apartado anterior. Dentro del proceso de deconstrucción del orden social, concretamente, se han empleado herramientas de investigación social como la observación participante, el análisis documental, los relatos de vida y las entrevistas en profundidad. De este modo, se han analizado los distintos fenómenos sociales que les acontecían entorno a las temáticas seleccionadas.

Master Vale Callar forma parte del proyecto que hemos denominado «Del becariado al precariado», diseñado por el Aula Laboratorio para prevenir y sensibilizar sobre temas relacionados con el ámbito del trabajo y que incluye la precariedad laboral a través de la representación de escenas cotidianas de precariedad en el ámbito laboral y académico basadas en fragmentos de historias de vida del alumnado universitario. En todo el proceso conectamos con la creación artística a través de imagen, sonido y palabra con los aportes de las ciencias sociales, lo que permite el diálogo y la reflexión comunitaria

${ }^{6}$ La cocreación de la pieza de Teatro INTERSOC con sustrato de Teatro de la Escucha: «Master Vale Callar», es resultado de un programa compuesto por una sesión semanal desarrollada los miércoles de 11 a $14 \mathrm{~h}$ durante cuatro meses abarcando veinticuatro horas de proceso creativo, más ensayos y representaciones, configurando un total doce sesiones. Para más información, consultar: https://www.youtube.com/watch? $\mathrm{v}=\mathrm{oEV} 3 \mathrm{k} 9 \mathrm{Q}-9 \mathrm{zc}$ 
para contribuir a visibilizar dicha precariedad laboral con el fin de contribuir a transformar la realidad social ${ }^{7}$.

En esta creación teatral colectiva se abordan los conflictos de precariedad laboral, patriarcado y acoso que padece el alumnado. Durante todo este proceso se aplica un método con perspectiva de género y técnicas cualitativas-participativas con gran aporte etnográfico (uso del cuaderno de campo, observación, recopilación documental, relatos de vida, video, fotografía, etc.). Una propuesta que tiene su humus en el Teatro Popular, Teatro del Oprimido (Teatro Foro/Teatro Imagen) y en el Teatro de la Escucha de Moisés Mato.

Master vale callar es una pieza de teatro INTERSOC creada de forma colectiva por el grupo de estudiantes, o población afectada, y apoyada por el grupo motor, a través de experiencias personales consensuadas del alumnado, comunes y reconocibles que trata de una estudiante universitaria de maestría de ciencias sociales:

Carolina, una chica de 23 años, de clase media, tradicional, de provincias y la menor de tres hermanos. Los dos mayores son varones. De padre funcionario y madre ama de casa. Gracias a su madre va a estudiar a Madrid, a casa de una amiga de su madre que está divorciada y vive con su hija. Ésta alquila habitaciones a estudiantes para sacar algo de dinero y así complementar sus ingresos. La pieza teatral nos va mostrando la precariedad vital de Carolina hasta llegar a la escena de la crisis, que hace una referencia específica a su necesidad de competir por las escasas becas existentes. Se normalizan situaciones que, vistas de forma individualizada, invisibilizan procesos de acoso ambiental subsumidos por la precariedad vital y laboral del alumnado universitario. En concreto, la creación colectiva de la dramaturgia hace referencia a procesos vividos por el alumnado participante que narran sus experiencias a la hora de solicitar becas. Estos relatos han servido para mostrar la realidad que vive Carolina, a nivel objetivo y subjetivo, mientras el departamento correspondiente hace la selección de becarios o becarias. Lo destacable es que son conocidos los actos de acoso sexista a las estudiantes becadas lo que genera que estas vivan esta realidad con gran estrés. Carolina, tras varios actos de acoso ambiental, se plantea el seguir o no con la beca, así como si denunciar o no los hechos vividos. Toda esta situación genera una situación de precariedad vital que le hace plantearse si el Master le vale callar o denunciar.

${ }^{7}$ La obra contó para su creación con la financiación, espacios y recursos materiales que tenía el Aula Laboratorio dados por la Facultad de Ciencias Políticas y Sociología de la Universidad Complutense de Madrid. El Aula Laboratorio está abierto a todos los y las estudiantes de la UCM, incluidos estudiantes Erasmus y visitantes con los que se ha contado en todas las ediciones. Es necesario decir que la mayor parte de los alumnos y las alumnas participantes pertenecían al ámbito de las ciencias sociales. 
No hay ninguna otra institución social, como el trabajo y las relaciones de género, para mostrar la potencia fetichizante del ya citado individualismo metodológico (que constituye el corpus teórico de referencia del orden liberal): su capacidad para mostrar las determinaciones estructurales que guían o condicionan las acciones de los actores sociales como si fueran sus preferencias. La precariedad laboral y la desigualdad de género son, en este sentido, dos de los principales estructurantes estructurados de la vida social en la modernidad. En el caso de la precariedad laboral, más allá de la degradación de las condiciones de vida y de trabajo que la acompañan, ésta produce también un debilitamiento de la potencia de subjetivación de los trabajadores, y sobre todo de las trabajadoras, potencia de subjetivación que daría lugar a formas de movilización susceptibles de frenar el proceso de precarización. Si son precarios/as, es porque no se movilizan; si están subordinadas, es porque no se empoderan. Estas son algunas de las premisas del discurso del poder.

Desde el Aula Laboratorio hemos constatado que el teatro INTERSOC es una herramienta que permite poner en circulación entre las personas participantes —actores, actrices, autores, autoras, espectadoras y espectadores-, estudiantes universitarios y universitarias en este caso, estas dos formas tautológicas de culpabilización de la víctima, apelando a su capacidad de transformación de la estructura social (Villasante, 2014), y descubrir realmente que lo que hacen es neutralizar la acción del sujeto como agente.

Es interesante destacar que en las diferentes interacciones del público con los actores y actrices no se logran transformar las estructuras de dominación que determinan el destino de los personajes, lo que en términos de género, por ejemplo, llama a la reflexión y reevaluación de los procesos de empoderamiento. No obstante, su importancia real no está en el producto final de esas interacciones, sino que radica en el hecho de la toma de conciencia de haber asistido a una transformación de los límites de la acción social que se sitúan, por otro lado, en los límites de la representación para dar cuenta de la acción social misma. Dicho de otra manera, el reconocimiento y superación de las individualidades, así como el reconocimiento de los problemas estructurales que conlleva el sistema y que son colectivos al grupo, a la comunidad y/o la sociedad, no sólo son fuente de resistencia individuales y/o colectivas, sino también de actos de empoderamiento individuales y colectivos promovidos en el foro por el debate y la reflexividad, siendo de gran importancia para el reconocimiento y afinidades electivas como grupo en la transformación social.

En este sentido, y dentro del reconocimiento de las limitaciones que puede tener la técnica empleada, así como sus herramientas, el teatro de intervención sociológica invita a revisar su metodología para incorporar en el proceso participativo nuevos 
elementos que conlleven a una transformación social consciente del problema planteado para alcanzar una inteligencia y acción colectiva a través de dispositivos que integren valores y emociones, lazos afectivos, para el empoderamiento y resistencia del grupo o comunidad afectada. Procesos que posteriormente serán analizados sociológicamente en una dinámica de investigación, acción, transformación, investigación. El teatro INTERSOC invita a buscar soluciones a la reflexión y facilita la comprensión de la realidad social a través de la interacción como sujeto-sujeto en la investigación.

\section{A MODO DE CONCLUSIÓN}

El teatro INTERSOC es muchas cosas, pero por encima de todo es nuestro propio enfoque y aproximación al Teatro Social. Partiendo de la elección del asunto a trabajar y continuando con el diseño colectivo del espectáculo como forma de autoconocimiento, empoderamiento y su posterior difusión como forma de reivindicación y transformación. El teatro INTERSOC del Aula Laboratorio intenta romper con las metodologías tradicionales académicas de enseñanza de las ciencias sociales, por otra más motivadora, comprensiva y transformadora para el estudiante universitario que, dentro de la lógica pedagógica, puede tener un efecto multiplicador y de desborde (Villasante, 2014) para la sociedad.

Como hemos visto, la implementación de técnicas y herramientas del Teatro Foro y el Teatro de la Escucha se ajusta adecuadamente a un marco de Investigación Acción Participativa. Lo que nos diferencia, desde el Teatro INTERSOC, de los otros tipos de teatros sociales, es que hay una reflexión sociológica anterior, durante y posterior a todo el proceso en el marco de la IAP. Por eso, no solo buscamos comprender y transformar, también buscamos crear conocimiento científico, pero no en el sentido hegemónico de la neutralidad suscita del poder, sino un conocimiento científico consciente de sus parcialidades (Villasante, 2006) o de conocimiento situado (Haraway, 1995). Este artículo es un ejemplo de ello.

En la misma génesis del proyecto se parte de la idea de promover la reflexión y el conocimiento mutuo entre las diferentes agencias implicadas con una perspectiva que permita el empoderamiento y la transformación efectiva en última instancia a través de la socialización del resultado en muestras públicas que nos permitan avanzar en la complejidad del sistema y, como en la pieza concreta analizada, avanzar en la deconstrucción de la precariedad laboral académica, así como del acoso sexual y sexista de la comunidad universitaria. 


\section{BIBLIOGRAFÍA}

Alberdi, I. (1999). «El significado del género en las ciencias sociales», en Política y sociedad, núm. 32, UCM, Madrid, pp. 9-21.

Amoros, C., ValcarcelL, A., y Camp, V. (1994). El concepto de igualdad. Editorial Pablo Iglesias.

Andrés, M.A (2005). «Propuesta indicadores del procesos enseñanza aprendizaje» en Relieve, pp. 63-82.

Arias Fernández, M. A. (2016). «La Trayectoria Histórica de la Sociología Cualitativa en España: La Influencia de Jesús Ibáñez». Investigación Cualitativa 1(2). 6-22. http://ojs.revistainvestigacioncualitativa.com/index.php/ric\%0A22.

Bauman, Z. (2004). Modernidad líquida. Buenos Aires, Fondo de Cultura Económica.

Beltrán, M. (1985). «Cinco vías de acceso a la realidad social», en Reis, pp. 7-41.

Benjamin, W. (2012). Angelus Novus, Comares, Granada.

Boal, A. (1974). Teatro del Oprimido y otras estéticas políticas. Buenos Aires. Ediciones de la Flor.

- (1982). Teatro del oprimido. Teoría y práctica. Nueva Imagen. México

- (2001). Juego para actores y no actores. Alba Editorial. Barcelona.

- (2004). Las técnicas latinoamericanas de teatro popular. Una revolución Copernicana al revés. Buenos Aires, Ediciones Corregidor.

- (2012). La Estética Del Oprimido. Barcelona: Alba.

Bourdieu, P., Passeron, C., \& Chamboredon, J. [(1973) 2002]: El Oficio del Sociólogo. México: Siglo XXI Editores. Colección Sociología y Política.

Bourdieu, P. (2007). La dominación masculina. Barcelona, Editorial Anagrama.

Calderón, J.A. y López Calle, P. (2014). «De la représentation de l'action et de l'action de représenter : contre les rigueurs du destin en sociologie». En José-Angel Calderon, Valérie Cohen (dir.), Qu'est-ce que résister? Usages et enjeux d'une catégorie d'analyse sociologique, Villneuve d'Ascq, Presses universitaires du Septentrion, col. «Le regard sociologique», pp. 145-167

Calsamiglia, A. y Cubells, J. (2016). El Potencial del Teatro Foro como Herramienta de Investigación. Athenea Digital, 16(1), 189-209. http://dx.doi.org/10.5565/rev/athenea.1462 [consultado en noviembre 2017].

Carnacea, Á. (2013). «El Tetuán de Ayer y de Hoy: Teatro, Diálogo Intergeneracional y Memoria.» In Arte, Intervención y Acción Social. La Creatividad Transformadora, eds. Ángeles Carnacea Cruz and Ana Lozano Cámbara. Madrid: Grupo 5, 377-386. Castillo, J.J. (2012). Clásicos y modernos en Sociología del Trabajo. Buenos Aires, Miño y Dávila. 
- (1994). El trabajo del sociólogo. Madrid, Ed. Complutense

- (2009). «Seguir a los clásicos: un taller oculto en la formación del sociólogo», en Política y Sociedad, vol. 46, núm. 3, pp. 77-90.

Castro, A. y González, A. (2008). «Psicodrama», en Seis Enfoques Piscoterapéuticos, Editorial El Manual Moderno, 187-252.

Castro, I. D. y Martín, P. (2012). El Desarrollo de la Inteligencia Emocional a Través Del Teatro, Para Promover Bienestar y Respeto a La Diversidad. Santander: V Congreso Mundial de Estilos de Aprendizaje, Santander, 27, 28 y 29 de junio de 2012.

Cerdeira, I (2017). Novelas e historias de vida. Asturias, Ed. Sapere Aude.

Colectivo IOE (1993). Metodologías participativas en la investigación social: 19-29 abril 1993. Madrid: CIMS.

De Mingo, A. (2012). «La comunidad en cuestión. Un episodio de la historia moral del teatro, según Rousseau», en Valenciana, núm. 9, enero-junio, 2012, pp. 91-119.

De Vicente, C. (2013). La escena constituyente. Teoría y práctica del teatro político, Madrid, Centro de documentación crítica.

Díaz, M. J. y García, F. (2018c). «Una mirada desde la Sociología Actual: análisis y propuestas del contexto social». Madrid: Editorial AMS.

Díaz, M. J. y Pastor, I (2018a). Del becariado al precariado. Análisis de la precariedad laboral del alumnado universitario a través de metodologías participativas en «Investigación y prácticas sociológicas: Escenarios para la transformación social». Madrid: Editorial UNED.

- (2018b). Innovar en nuevas metodologías para el aprendizaje: el aula-laboratorio de teatro social en «Una mirada desde la Sociología Actual: análisis y propuestas del contexto social». Madrid: Editorial AMS.

Eco, U. (1992). Obra abierta. Barcelona, Editorial Planeta-Agostini [https://direccionmultiple.files.wordpress.com/2012/08/eco_umberto-obra_abierta.pdf].

Esteban, I. G., y Fernández, E. A. (2017). Fundamentos y técnicas de investigación comercial. Madrid, ESIC Editorial.

Fàbregues, S., Meneses, J., Rodríguez, D. y Paré, M. (2016). Técnicas de investigación social y educativa. Barcelona, Editorial UOC.

Francés, V.; Gálvez, A.; Izquierdo, A.; Kuric, S.; Laforgue, N.; Marano, C.; Matos, O.; Rodríguez, S.; Rubio, A. (2016). Repensando nuestra ciudad. Madrid: Centro Reina Sofía sobre Adolescencia y Juventud, Fad. DOI: 10.5281/zenodo.3653760

Francés, F. J. (2016). Metodologías participativas para la investigación y la intervención social. San Vicente del Raspeig, Publicaciones de la Universitat d'Alacant, D. L.

Freire, P. (1970 [2009]). Pedagogía Del Oprimido. Madrid: Siglo XXI. 
Friedman, D. (2016). «And Development The Performance Activism of the Castillo Theatre». The Drama Review 4: 68-91.

García, F. y Díaz, M.J. (2018a). «Investigación y prácticas sociológicas: escenarios para la transformación social». Madrid: Editorial UNED.

- (2018b). La investigación sociológica para conocer los escenarios y las dinámicas de la transformación social, en «Investigación y prácticas sociológicas: Escenarios para la transformación social». Madrid: Editorial UNED.

García-Huidobro, V. (2008). «Pedagogía Teatral: Aporte Para Estudiantes y Docentes». Educadores, 7.

Gaulejac, V. (2016). Las fuentes de la vergüenza. Madrid, Colección Sociología Clínica. Haraway, D. (1995). «Conocimientos situados: la cuestión científica en el feminismo y el privilegio de la perspectiva parcial», en Ciencia, ciborgs y mujeres. La reinvención de la naturaleza, Madrid: Cátedra, pp. 313-346.

Hayes, P. Cantillon, P. (2014). «Discovering Emotional Honesty through Devised Theatre». The Clinical Teacher 11: 84-87.

Hidalgo, M.A. (2011). «Apuntes Sobre Arteterapia y Aplicaciones Del Arte Para El Diálogo y La Integración Social», en Arte, Intervención y Acción Social. La Creatividad Transformadora, eds. A. Carnacea and A. Lorenzo. Madrid: Grupo 5, 141143.

Ibáñez, Jesús. (1986). «Perspectivas de La Investigación Social: El Diseño En Las Tres Perspectivas», en El Análisis de La Realidad Social. Métodos y Técnicas de Investigación Social, eds. Manuel García Ferrando, Jesús Ibáñez, and Francisco Alvira. Madrid: Alianza, 57-98.

Junker, B (s.f.). Introducción a las ciencias sociales. El trabajo de campo. Ediciones Marymar.

Kuric, S. (2020). Artesanía Teatral: modelos de creación, organización y condiciones sociolaborales desplegadas en el panorama teatral madrileño. [Tesis Doctoral]. https://eprints.ucm.es/59139/

Lagarde, M. (1996). Género y feminismo. Desarrollo humano y democracia. Madrid, Horas y Horas.

Lewin, K. (1947). Frontiers in Group Dynamics. Tavistok: SAGE Publications.

Liamputtong, P. y Rumbold, J. (2008). Knowing Differently: Arts-Based and Collaborative Research Methods. Nueva York: Nova Science Publishers.

Lillo, F. G. (2013). «Aprendizaje Colaborativo en la Formación, Universitaria de Pregrado» en Revista de Psicología, Universidad Viña del Mar, 2013, vol. 2, n. ${ }^{\circ}$ 4, 109 142. 
López Fernández, M. (2011). «Cómo Hacer Una Sopa Con Piedras: El Arte Como Herramienta de Intervención y Mediación Social. Construyendo Sociedades Más Creativas», en Arte, Intervención y Acción Social. La Creatividad Transformadora, eds. Ana Carnacea and Ana Lozano. Madrid: Grupo 5, 97-129.

Madurga, A. y Cubells, J. (2016). «El Potencial Del Teatro Foro Como Herramienta de Investigación». Athenea Digital. Revista de pensamiento e investigación social 16(1). 189-209.

Marugán, B. y Díaz, M.J. (2015). «Madrileñas ante la crisis: trabajar, trabajar y trabajar», en Lucas Marín, A. y Cáceres Zapatero, M. Madrid ante los desafios sociales actuales. La realidad social de Madrid Vol.III, Editorial Fragua, Madrid.

Mato, M. (2016). Reflexiones sobre el Teatro Foro desde el Teatro de la Escucha. Madrid, Sala Metáforas.

Mayo, E. (1945). The Social Problems of an Industrial Civilization. Boston: Harvard University.

Montañés, M. (2009). Metodología y técnica participativa. Barcelona, Editorial UOC.

Morán, J. T., Ruiz, M.E. y Marín, D. (2016). «El Teatro, un recurso para el desarrollo de competencias y para la inclusión». Revista Aula. Prácticas de aula y de centro, 248: 39-43.

Motos-Teruel, T. Y Benlliure, V. (2018). «Beneficios de Hacer Teatro En El Desarrollo Positivo En Adolescentes de Valencia». Revista de Investigación en Educación 16(1). 34-50.

Munro, M, Munro, A. Lemmer, K. y Pretorius, M. (2015). «Theatre Strategies to Develop Emotional Intelligence Skills in Business Communication: An Exploratory Study». Southern African Business Review 19(2). 1-26.

Park, P. (1992). «Qué Es La Investigación-Acción Participativa. Perspectivas Teóricas y Metodológicas», en La Investigación Acción Participativa. Inicios y Desarrollos, Ed. Popular, Madrid, ed. María Cristina Salazar. Madrid: Editorial Popular, 135174.

Pereda, C. y de Prada, M.A. (2015). «Investigación Acción Participativa y Perspectiva Dialéctica». Arxius: 3-15.

Pereda, C. y de Prada, M.A. y Actis, W. (1997). Voluntariado y Democracia Participativa. Reflexiones a partir del «Proyecto $+60 »$, Investigación Acción Participativa en el barrio de Prosperidad (Madrid). Madrid. https://www.colectivoioe.org/ uploads/62145a362f7e337cbdbdc6e873e991146cf40b1d.pdf

- (2003). «Investigación-Acción Participativa: Propuesta Para Un Ejercicio Activo de La Ciudadanía», en Encuentro de La Consejería de Juventud, 1-26. http://www. 
monografias.com/trabajos32/investigacion-accion-participativa-ejercicio-ciudadania/investigacion-accion-participativa-ejercicio-ciudadania.shtml

Pizzorno, A. (1987). «Politics unbound», en C. Maier (ed.), Changing Boundaries of the Political: Essays on the Evolving Balance between the State and Society, Public and Private in Europe (Cambridge Studies in Modern Political Economies, pp. 27-62). Cambridge: Cambridge University Press.

Preston, S. (2013). «Research in Drama Education : The Journal of Applied Theatre and Managed Hearts? Emotional Labour and the Applied Theatre Facilitator in Urban Settings». Research in Drama Education: The Journal of Applied Theatre and Performance, 18(3). 230-245.

Sanchis, E. (2016). Los parados. Cómo viven, qué piensan, por qué no protestan. Valencia: Universitat de València.

Santos, B. (2017). Teatro del Oprimido. Raíces y alas. Una teoría de la praxis. Editorial Descontrol.

Scholz, R. (2013). «El patriarcado productor de mercancías. Tesis sobre capitalismo y relaciones de género», en Constelaciones. Revista de Teoría Crítica, vol. 5. ISSN 2172-9506. http://constelaciones-rtc.net/article/view/815/869

Standing, G. (2011). El precariado. Una nueva clase social. Barcelona, Pasado \& Presente.

Vieites, M. F. (2013). «La construcción de la pedagogía teatral como disciplina científica». Revista Española de Pedagogía (256). 493-508.

- (2016). «Trabajo Social y Teatro: Considerando Las Intersecciones.» Cuadernos de Trabajo Social, 29(1). 21-31.

Villasante, T. (2006). La socio-praxis: un acoplamiento de metodologías implicativas. Metodologías de investigación social. Introducción a los oficios, 379-405.

- (2010). «Reflexividades Socio-Práxicas: Esquemas Metodológicos Participativos.» Cimas Cuadernos 2(2). 2-25.

- (2014) Redes de vida desbordantes. Fundamentos para el cambio desde la vida cotidiana. Madrid: Editorial Catarata.

Willis, P (1988). Aprendiendo a trabajar. Cómo los chicos de clase obrera consiguen trabajos de clase obrera. Madrid, Ediciones Akal.

Yzaguirre, F. y Castillo, C.A. (2013). «La perspectiva de la sociología clínica: una sociología de proximidad orientada al sujeto», en Actas del XI Congreso Española de Sociología: crisis y cambio, propuestas desde la sociología, volumen ADENDA, pp. 832-840. 